\title{
Der Bolognaprozess und die europäische Wissensgesellschaft
}

\author{
Von Kathia Serrano-Velarde
}

Zusammenfassung: Der Artikel geht der Frage nach, wie sich die „Wissensgesellschaft“ zum ,enjeu politique' europäischer Reformdiskurse entwickelte. Am Beispiel des Bolognaprozesses soll die (bildungs)politische Umdeutung des Begriffes expliziert, seine Operationalisierung zum implementierbaren Reformkonzept nachvollzogen und seine Umsetzung im Reformprozess diskutiert werden. Was für eine Vision der Wissensgesellschaft wird in den Policy-Texten des Bolognaprozesses beschrieben? Wo liegen die Möglichkeiten und Grenzen zur Realisierung eines solchen Gesellschaftsmodells?

\section{Einleitung}

In den Sozialwissenschaften herrscht wenig Einigkeit über die definitorischen Merkmale der Wissensgesellschaft ${ }^{1}$. Zwar herrscht Konsens darüber, dass Wissen (insbesondere wissenschaftliches Wissen) die Gesamtheit der sozialen Systeme durchdringt bis hin zur individuellen Lebensführung, die sich den stetig wachsenden Wissensmengen und einer komplexer werdenden Umwelt anpassen muss. Bei der Interpretation von Wirkungsmechanismen und -effekten wissensbasierten Wandels scheiden sich aber die Geister. Auch die Frage, ob das Konzept der Wissensgesellschaft als Etikett für die Gegenwartsgesellschaft auf etwas genuin Neuartiges verweist, ist ein zentraler Diskussionspunkt sozialwissenschaftlicher Debatten (Maasen / Weingart 2000): War die Gesellschaft nicht von jeher wissensbasiert? Brachte nicht jedes Kapitel gesellschaftlicher Entwicklung seine ihm eigenen Formen der Wissensproduktion, -diffusion und -tradierung hervor (Heidenreich 2003; Webster / Robins 1989; Webster 2000)?

Der analytische Zuschnitt der Forschungsansätze auf einen bestimmten Moment des sozialen Wandels ermöglicht eine Differenzierung der wissensbasierten Gesellschaftsmodelle. So versucht sich Fritz Machlup (1962) beispielsweise an einer quantitativen und ökonomisch geleiteten Analyse der expandierenden „,knowledge industries“, während Daniel Bells (1976) evolutionistisches Gesellschaftsmodell auf die Prämisse zurückzuführen ist, dass Arbeitsmodi und Arbeitskräfte einem kontinuierlichen Rationalisierungsdruck ausgesetzt sind, der zum Rückgang handwerklicher zugunsten wissensbasierter Arbeitsformen führt. Im Werk Manuel Castells (1996) stehen wiederum die organisatorischen und kulturellen Implikationen der Computer- und Netzwerktechnologie im Mittelpunkt der Analyse.

Es ist sowohl der Abstraktheit als auch der Schwierigkeit, die postulierten Gesellschaftsmodelle empirisch zu fassen, zuzurechnen, dass dem Begriff der Wissensgesellschaft ein hohes $\mathrm{Ma} ß$ an Verschwommenheit anhaftet. Als Vision und abstraktes Gesellschaftsmodell erzeugt die Wissensgesellschaft große Resonanz in der außerwissenschaftlichen Öffentlichkeit. Was durch den inflationären Gebrauch dieses Gesellschaftslabels jedoch verdeckt wird, ist die politische Dimension, die jede prominente Zeitdiagnose enthält: „In ihnen spiegelt sich der öffentliche Kampf um die legitime Sicht auf die Welt. Die in Zeitdiagnosen versteckten oder offenen Klassifikationsprinzipien präjudizieren den Bereich des Denkbaren ebenso wie den des Undenkbaren und der Denkverbote“ (Bittlingmayer 2005: 17). Daher mag es nicht verwundern, dass gerade der Begriff der Wissensgesellschaft immer wieder von politisierten Fortschrittsrhetoriken eingeholt wird. Dieses Phänomen lässt sich mitunter auch in bildungs-

1 Hiermit möchte ich Cécile Cuny, Sezin Topçu und den anonymen Gutachtern des Artikels für ihre Kommentare danken.

Soziale Welt 60 (2009), S. $339-352$ 
und forschungspolitischen Diskursen feststellen. Tatsächlich ist die Wissensgesellschaft zum ,enjeu politique' der Europäischen Union (EU) promoviert worden. Auch der Bolognaprozess zur Realisierung eines gemeinsamen europäischen Hochschulraumes bedient sich dieser Rhetorik, um seine Reformagenda zu kontextualisieren. Der Bolognaprozess wurde 1998 in Paris aus der Taufe gehoben und zielt darauf ab, eine Angleichung europäischer Hochschulstrukturen einzuleiten, die studentische Mobilität zu fördern und die Vergleichbarkeit akademischer Abschlüsse zu gewährleisten. Die damalige Erklärung der französischen, britischen, italienischen und deutschen Bildungsminister (Sorbonne Declaration 1998) setzte einen Reformprozess in Gang, dem sich über die letzten neun Jahre mehr als 40 Mitgliedstaaten angeschlossen haben und der alle zwei Jahre zur Formulierung von detaillierten Reformprinzipien zu Studienstruktur, Studienabschluss, Studienverlauf und Qualitätssicherung führte.

Im Folgenden soll es nun darum gehen, das wissensbasierte Gesellschaftsmodell zu explizieren, das diesem Reformprozess zugrunde liegt, seine Wirkungsmechanismen zu erläutern und schließlich den Inhalt und die Essenz der europäischen Reformagenda kritisch zu durchleuchten: Was für eine Vision der Wissensgesellschaft wird in den Policy-Texten des Bolognaprozesses beschrieben? Wo liegen die Möglichkeiten und Grenzen zur Realisierung eines solchen Gesellschaftsmodells auf europäischer Ebene? Im Mittelpunkt des Forschungsinteresses steht nicht etwa die Übersetzung eines wissenschaftlichen Begriffs in den politischen Diskurs. I Vielmehr handelt es sich darum, einen bildungspolitischen Entwurf der Wissensgesellschaft zu untersuchen und kritisch zu diskutieren, der seit nunmehr zehn Jahren in den europäischen Politikforen zirkuliert und weitläufige, hochschulpolitische Reformen mit begründet hat.

Die Studie fokussiert vornehmlich auf die Meta-Ebene des politischen Geschehens, d.h. auf den europäischen Policy-Making-Prozess. Unter dem Begriff des Policy-Making wird die Konzeptualisierung und Ratifizierung des europäischen Reformprogramms und der dazugehörigen Policy-Texte verstanden (Muller 1990). Die Untersuchung basiert auf einer thematischen Inhaltsanalyse (Froschauer / Lueger 2003) der Policy-Dokumentation Bolognas sowie auf der Auswertung 70 semi-struktuierter Interviews mit Zeitzeugen und Akteuren des europäischen Policy-Prozesses.

Der Artikel ist in vier Abschnitte gegliedert: Im Anschluss an diese Einleitung sollen die beiden wichtigsten Versionen der Wissensgesellschaft für den europäischen Hochschulbereich dargestellt und verglichen werden. Ein besonderes Augenmerk liegt hierbei auf der Inklusionsdynamik, die das Bolognamodell der Wissensgesellschaft charakterisiert. Diese Dynamik betrifft erstens die Inklusion breiter Bevölkerungsschichten in ein wissensdominiertes Gesellschaftsmodell über die Rationalisierung des Wissenserwerbs; zweitens greift die Inklusionsdynamik bis in die individuelle Lebensführung hinein, insofern sich das Individuum sein Leben lang dem Erwerb neuer Kenntnisse verschreibt (Stichwort: Lebenslanges Lernen); drittens hat der Bolognaprozess eine bildungspolitische Plattform ins Leben gerufen, die sich durch die Inklusion neuer Interessengruppen in die Erarbeitung und Umsetzung der Reformrichtlinien charakterisiert. Es wird argumentiert, dass das Bologna-Modell der Wissensgesellschaft auf dieser dreifachen Inklusionsdynamik aufbaut. In einem letzten Punkt werden schließlich die Möglichkeiten und Grenzen des Bolognamodells der Wissensgesellschaft analysiert und diskutiert.

\section{2. „European knowledge economy“ oder „Europe du Savoir“: Bildungspolitische Dimensionen der europäischen Wissensgesellschaft}

Es sollte grundsätzlich zwischen zwei verwandten visionären Ansätzen in der europäischen Bildungspolitik unterschieden werden: der in Lissabon lancierten EU-Agenda, die zum Ziel hat, ,die Union zum wettbewerbsfähigsten und dynamischsten wissensbasierten Wirtschafts- 
raum der Welt zu machen“ (Europäischer Rat 2000) einerseits und dem Bekenntnis europäischer Bildungsminister zum „Europe du Savoir“ (Sorbonne Declaration 1998) andererseits. Diese Unterscheidung ist umso bedeutsamer, als sich das intergouvernementale Bologna-Projekt zumindest diskursiv und im Namen des Subsidiaritätsprinzips von EU-Initiativen in verwandten Politikfeldern - insbesondere in der Forschungspolitik, in der die EU-Kompetenzen durch die Einrichtung eines Europäischen Forschungsrates und den dazugehörigen Fördertöpfen ausgeweitet worden sind - absetzt und in Konkurrenz zu ihnen tritt.

\section{Lissabon und die Regulierung der Wissensproduktion}

Als unerschöpfliche Ressource nimmt Wissen einen zentralen Platz in der Wirtschaftsplanung des Produktionsstandorts Europas ein. ${ }^{\text {II }}$ Diese Strategie geht nicht zuletzt auf die prominenten Schlussfolgerungen der Lissabonner Regierungskonferenz zurück, Europa bis 2010 zum wettbewerbsfähigsten wissensbasierten Wirtschaftsstandort der Welt zu entwickeln.

Das EU-Konzept der so genannten „knowledge economy“ basiert auf der systemtheoretisch informierten Überzeugung, dass durch die Integration des Wissenschafts- und Bildungssystems, des ökonomischen und politischen Systems produktive Synergieeffekte freigelegt werden, die Europa aus der wirtschaftlichen Stagnation herausbewegen könnten. Die erhofften Synergieeffekte erfordern jedoch auch eine neue Art der Steuerung, eine staatliche Regulierung, die nicht länger als Restriktions- sondern Ermöglichungsstruktur auftritt (Willke 2007, 1997). Die Lissabonner Strategie sollte daher als umfassendes Policy-Paket verstanden werden, in der die Wissensgenerierung und -produktion einen prominenten Platz einnimmt. Die Lissabonner Agenda umfasst sowohl den Wissenschaftsbereich als auch mikro- und makroökonomische Reformen, Förder- und Richtlinien zur Informationstechnologie (IT) sowie Bildungs- und Sozialfragen. Im Rahmen dieser Aktivitäten werden europaweit geltende Richtlinien vorgelegt, ,benchmarking' und Evaluation betrieben und schließlich auch themenspezifische Partnerschaften ins Leben gerufen. Prinzipiell handelt es sich also um die gleichen Policy-Instrumente, die auch im Bologna-Prozess Verwendung finden, mit der offensichtlichen Ausnahme, dass die Europäische Kommission federführend in die Politikformulierung und -umsetzung eingreift. Relativierend sollte hinzugefügt werden, dass sich der Erfolg dieser Strategie in Grenzen hält. So haben sich die EU-Mitgliedsstaaten in Lissabon zwar dazu verpflichtet, die Bildungsausgaben auf drei Prozent des jeweiligen Bruttoinlandprodukts zu erhöhen. Tatsächlich stagnieren die Ausgaben für die Schul- und Hochschulausbildung seit Jahren, wenn sie nicht sogar sinken (Kok 2004). Des Weiteren belegen die Studien von Jan Servaes eine zunehmende Diskrepanz (oder auch „knowledge gap“ / „digital divide“; Sevraes 2005) zwischen den Bevölkerungsteilen, die sich die neuen IT-Medien leisten können, und jenen, die aus Bildungs- oder finanziellen Gründen aus dem so genannten „e-Europe“ ausgeschlossen sind.

\section{Bologna und die Regulierung des Wissenserwerbs}

Mit der Fixierung auf die Wiederverwertbarkeit von Wissen für die Produktionsverhältnisse im europäischen Binnenmarkt kontrastiert der ideologische (Norval 2000) Ansatz der BolognaErklärung. III Besonders in den frühen Reformerklärungen schwingt eine deutliche sozialpolitische Komponente in der Politikformulierung mit: Hier geht es um die Inklusion (Luhmann 1995) des ,citoyen européen' in ein gesellschaftspolitisches Weltbild, da er nur als Wissender am sozialen Wohlstand teilhaben und neue Formen der demokratischen Partizipation ausleben kann:IV „A Europe of knowledge is now widely recognised as an irreplaceable factor for social and human growth and as an indispensable component to consolidate and enrich the European citizenship, capable of giving its citizens the necessary competences to face the challenges of the new millennium, together with an awareness of shared values and belonging to a common 
social and cultural space" (Bologna Declaration 1999: 1). Das Inklusionsmodell der BolognaWissensgesellschaft geht von einem Wissensbegriff aus, der die Wissensressource als Handlungskapazität begreift. Bezieht man sich auf die Ausführungen Nico Stehrs, so definiert sich die Handlungskapazität von Wissen darüber, dass Wissen die Wirklichkeit verändern kann: „Knowledge as capacity of action implies that access to knowledge is constitutive for the operational scope of the individual. The special importance of scientific and technical knowledge, in any modern society, derives not so much from the fact that it is at times treated as if it is essentially uncontested (or objective) but that it constitutes, more than any other form of modern knowledge, an incremental capacity for social action or an increase in the ability of ,how to do it' which, moreover, may be privately appropriated, if only temporarily" (Stehr 1994: 97). Das Individuum eignet sich also Wissen an, damit es an den gesellschaftlichen Prozessen teilnehmen und seine ihm zustehenden Handlungsmöglichkeiten wahrnehmen kann. Allerdings setzt ein breiter Zugang zu Wissen als ökonomische, aber auch soziale und politische Ressource eine outputorientierte Regulierung des Wissenserwerbs voraus. Diese wird über implementierbereite Reformkonzepte umgesetzt.

\section{Implementierung eines wissensbasierten Gesellschaftsmodells? Bologna und die Rationalisierung von Wissensprozessen}

Aufgrund der stetig wachsenden zugänglichen Wissensmengen sowie der ökonomischen Notwendigkeit, innovatives Wissen in den Produktionsprozess einzubinden, postuliert Bologna eine Revision traditioneller Ausbildungswege. Schließlich müssen zukünftige Wissensarbeiter (Machlup 1962; Willke 1998) lernen, mit einer dynamischen Produktionsressource umzugehen. Weiterhin muss, um den Handlungs- und Produktionsspielraum vollends auszunutzen, die Möglichkeit des Wissenszuwachses in der Ausbildungsstruktur und im Prozess des Wissenserwerbs antizipiert werden. Ergo sollen nicht nur mehr Arbeiter auf den Markt kommen, die innovatives Wissen aufgrund ihrer wissenschaftlichen Hochschulausbildung rezipieren und verarbeiten können. Ihnen muss auch die Möglichkeit gegeben werden, ihre wissenschaftliche Ausbildung, wann immer sie es für notwendig erachten, fortzusetzen und / oder auszuweiten. Daher auch das zentrale Bekenntnis Bolognas zur Förderung lebenslangen Lernens. Wissenschaftliche Neugier wird zum Wirtschaftsmotor, insofern sich diese nicht nur auf Wissenschaftskreise beschränkt, sondern auch eine feste Größe im professionellen Selbstverständnis der europäischen Beschäftigten darstellt. Nur so lässt sich der wissensbasierte Produktionszyklus schließen. Hier ist also eine Inklusionsdynamik am Werk, die auf die Ausweitung des Lernprozesses auf die gesamte individuelle Lebensspanne abzielt. ${ }^{~}$

Als wissenschaftlich qualifizierte Arbeitskraft ist der europäische Wissensarbeiter des Weiteren am Prozess der Wissensgenerierung beteiligt. Objektiviertes, produktives Wissen muss nicht länger einzig und allein aus wissenschaftlichen Kreisen stammen, um Innovationen anzustoßen. Forschungs- und bildungspolitische Stellungnahmen betonen die wachsende Bedeutung und Legitimität angewandten Wissens, von Wissen also, das außerhalb des Wissenschaftssystems aus der Produktionspraxis heraus geboren und nachhaltig verwissenschaftlicht wird (Gibbons et al. 2004; Etzkowitz / Leydesdorff 1997). Der Übergang zwischen Wissenschaft und Produktionssystem gestaltet sich fließend unter der Voraussetzung, dass der Arbeiter in der Lage ist, diese Mediation zu bewältigen (siehe Abb. 1). Hierzu bedarf es, wie gesagt, einer gezielten Regulierung des Wissenserwerbs. 
Abbildung 1: Wissensbasierter Produktionszyklus

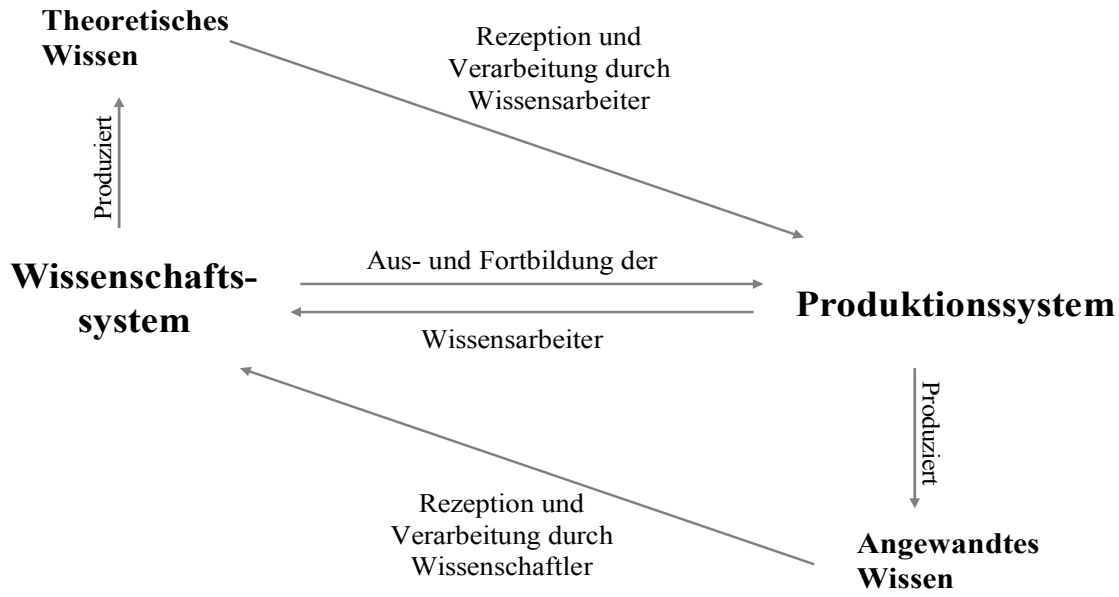

Die Policy-Dokumente des Bolognaprozesses stellen vorwiegend technische Richtlinien zur Studienstrukturierung bereit. Aus diesem Grund sollte eine Unterscheidung zwischen der Regulierung von Wissensinhalten einerseits (Curriculumentwicklung) und Wissensprozessen andererseits (Studienreform) eingeführt werden. Mit dem Begriff, Wissensprozess' ist die intellektuelle Aneignung von Wissensinhalten gemeint (Stehr 1994). Versucht man den Bolognaprozess im Lichte dieser Erkenntnis zu analysieren, wird deutlich, dass die stipulierte Umgestaltung der Studienstruktur, d.h. die Sequenzierung von Lernprozessen in drei Studienzyklen und einer bestimmten Anzahl von Modulen, sowie die Fixierung auf ,learning outcomes' eine tiefgreifende Veränderung akademischer Wissensprozesse begründet. Die Quantifizierung von Wissen und Wissensleistungen über das ECTS-Punktesystem (European Credit Transfer System) bildet den Ausgangspunkt und die Grundlage dieser „Wissenspolitik“ (Stehr 2003).VI Wissensmengen lassen sich nun mal besser verwerten und vergleichen als Wissensinhalte. Wissen wird dabei als , black box' gehandhabt, das einer kontextspezifischen Variation unterliegt und lokal, über nationale Qualitätssicherungsagenturen, kontrolliert werden soll. Auch hierfür stipuliert Bologna einheitliche Kontrollmechanismen. Der Wissensprozess wird durch die homogenisierte Sequenzierung des Wissenserwerbs in akkumulierbare Wissensmengen gesteuert. Die Studienstruktur wird flexibilisiert, damit das Individuum lebenslang einen unkomplizierten Zugang zu Weiterbildungsangeboten im Wissenschaftssystem erhalten kann. Die Verwertbarkeit einer Qualifikation, als provisorischer Abschluss eines Wissensprozesses, wird dabei von der Anschluss- und Ausbaufähigkeit der belegten Wissenskompetenz bestimmt. Hinter diesem Reformkonzept steht die geläufige Vorstellung, dass die Berechenbarkeit eines in Sub-Einheiten gegliederten Studiums die höhere Ausbildung auch für jene Individuen attraktiv macht, die bislang auf ein Studium aus Zeit- oder Kostengründen verzichten mussten.

Die Erklärungen des Bolognaprozesses unterscheiden, wie gesagt, zwischen der Homogenisierung von Wissensprozessen und der operationalen Diversität von Wissensinhalten. Wie aber lässt sich diese Fokussierung auf die Regulierung von Wissensprozessen erklären? In der Tat verlieren Wissensinhalte bzw. Bildungswissen in der europäischen Reformdiskussion zunehmend an Bedeutung. Dem Medienwissenschaftler Hans-Dieter Kübler zufolge hat ,[Bildungswissen] weder mit der kuranten Diskussion um Wissensgesellschaft unmittelbar und beeinflussend etwas zu tun, noch wird es innerhalb ökonomischer Verwertungskoordinaten als relevant erachtet" (Kübler 2004: 115). Dies mag der Tatsache geschuldet sein, dass es 
keinen eindeutigen europäischen Bildungskanon mehr gibt, an dem sich die beschriebene Inklusionsdynamik orientieren könnte: „Die humanistische Bildung, im 19. Jahrhundert der Horizont, der alle umschloss, die einen akademischen Beruf ausübten, Engländer, Russen Italiener, Juristen, Ärzte, Ingenieure, ist im Laufe des 20. Jahrhunderts untergegangen, hier früher, dort später, teils allmählich, teils in ruckartigen Schüben“" (Fuhrmann 2002: 32). Das europäische Kulturerbe bestünde demnach aus einer Ansammlung national abgrenzbarer, jedoch diffusionstauglicher Wissensströmungen und Werke, die heute unter dem Schlagwort, Allgemeinbildung' subsumiert werden, aber kaum mehr als Referenz für die individuelle Lebensführung oder für ein zukunftsträchtiges Gesellschaftsmodell europäischen Ausmaßes wahrgenommen werden könnten. Eine weitere Erklärung ist, dass Wissen selbst mit einem Unsicherheitsfaktor belegt ist: Was weiß man im Zeitalter des, information overload' überhaupt noch? Ist das, was wir wissen, reliabel? Wie steht es um die Gültigkeit von Wissen? Der Fokus liegt also nicht mehr auf dem Problem des ,Was muss ich wissen?', sondern vielmehr auf der Frage ,Wie gehe ich mit mehr oder weniger kurzlebigen Wissensinhalten um?'. Es liegt ein gewisser Pragmatismus im Reformansatz Bolognas: Wissensprozesse vermag man, im Gegensatz zu Wissensinhalten, leichter zu bewerten, zu erschließen. Sie eignen sich deshalb zur Reform. Aus diesem Grund - und weil es sich bei der Frage um Wissensinhalte immer noch um ein symbolisch aufgeladenes Subsidiaritätsproblem handelt - beschränkt sich die Reformagenda Bolognas vornehmlich auf die Regulierung von Wissensprozessen.

Im Grunde genommen verweist das Bologna-Modell der Wissensgesellschaft auf eine zweidimensionale Inklusionsdynamik: Zum einen geht es um die Inklusion einer größtmöglichen Anzahl von , citoyens européens' in den Prozess der Hochschulbildung als Conditio für ihre nachhaltige Einbindung ins und Partizipation am modernen Gesellschaftsmodell. Zum anderen wird die wissenschaftliche Aus- und Weiterbildung zum Leitmotiv individueller Lebensführung. Der Lernprozess umfasst die gesamte Lebensspanne eines Individuums. Durch seine Eingliederung in einen effizienten und flexiblen Prozess des Wissenserwerbs wird es für die Herausforderungen einer wissens- und wissenschaftsdurchdrungenen Gesellschaftsform konditioniert: Die Berechenbarkeit eines flexiblen und kumulierbaren Studiums soll die Inklusion bislang Ausgeschlossener in wissenschaftliche Ausbildungswege fördern. Außerdem soll die Flexibilität der Studienstruktur einen spontanen Zugang zu wissenschaftlichen Ausbildungsstrukturen ermöglichen, so dass ein Individuum sein Leben lang den Kontakt zur Alma Mater und somit zu innovativem, theoretischem Wissen aufrechterhalten kann. Im Gegensatz zu den bildungspolitischen Bestrebungen der Europäischen Union steht im Zentrum des Gesellschaftsbildes Bolognas der Mensch als Träger einer Wissensressource, nicht die abstrakte und verwertbare Wissensressource an sich. Nachdem nun zwei inhaltliche Aspekte der europäischen Wissensgesellschaft expliziert wurden, die in der Policy-Dokumentation ausgearbeitet werden, soll im folgenden Teil auf die Struktur des Politikprozesses eingegangen werden. Es wird argumentiert, dass die oben dargestellte Inklusionsdynamik ein strukturelles Pendant in der Entwicklung bildungspolitischer Richtlinien hat. Der Bolognaprozess zeichnet sich durch die Inklusion neuer Akteurs- und Interessengruppen in politische Entscheidungsprozesse auf supranationaler Ebene aus und führt so zu einem Paradigmenwechsel in der Gestaltung europäischer Bildungspolitik.

\section{Die Inklusion neuer Akteursgruppen in den europäischen Policy-Making- Prozess}

Seit der Bolognaerklärung im Jahre 1999 schlossen sich zahlreiche transnational organisierte Stakeholder, wie z.B. die European University Association (EUA), die European Student's Union (ESU), die European Association of Institutions in Higher Education (EURASHE) und die European Association for Quality Assurance in Higher Education (ENQA), dem PolicyMaking-Prozess an. Dank der flexiblen und offenen Policy-Struktur konnten diese Interes- 
sengruppen bereits sehr früh Interventionsmöglichkeiten im Rahmen der Politikformulierung wahrnehmen. Die Expertise und das ausschlaggebende Engagement der Stakeholder in der Erstellung der für den Bolognaprozess so zentralen Policy-Dokumentation haben deren Einfluss auf den Policy-Prozess etabliert:VII Als Daten- und Ideenlieferanten bestimmen sie den inhaltlichen Zuschnitt der Politikagenda und Reformansätze seit 2001 mit. Damals wurden europäische Interessengruppen im Rahmen der ersten ,Bologna Follow-up Group' aktiv. Diese informelle Gruppe wurde aus Vertretern der deutschen Regierungsdelegation und einigen Stakeholdern zusammengesetzt. Man traf sich in regelmäßigen Abständen zur Vorbereitung der Berliner Konferenz 2003 und des wegweisenden Kommuniqués. Aus dieser losen Kooperationsstruktur sollte alsbald eine Expertengruppe erwachsen, die sich systematisch mit Grundsatzfragen auseinander setzen und sich der Erstellung der Policy-Papiere (d.h. der Kommuniqués und ausgearbeiteter Reformkonzepte) annehmen sollte. Die 2003 einsetzende Verstetigung der ,Bologna Follow-Up Group' geht auf die sich zuspitzende Notwendigkeit zurück, dem alle zwei Jahre stattfindenden und expandierenden Policy-Prozess eine organisatorische Kontinuität zu verleihen. Durch ihr frühes Engagement in der Berliner Vorbereitungsgruppe konnten sich die Stakeholder allerdings eine wichtige Position in der Redaktion der PolicyTexte und Konferenztexte sichern. Bislang wächst die Zahl der im Bolognaprozess aktiven Interessengruppen kontinuierlich, ohne dass Überlegungen zur Einführung von Selektionsmechanismen stattfinden.

Die Inklusion neuer Akteursgruppen in politische Entscheidungsprozesse auf supranationaler Ebene ist bezeichnend für den Paradigmenwechsel europäischer Bildungspolitiken. Der Wunsch nach einem breit angelegten Diskussionsprozess schlägt sich auf nationaler Ebene mitunter in der Zusammenstellung gemischter Arbeitsgruppen (AG) nieder, die ministerielle und Interessenvertreter zusammenführen und mit einer Beraterfunktion für ausgewählte Fragestellungen versehen (das deutsche Bundesministerium für Bildung und Forschung hat eine solche Bologna-AG bereits 2003 ins Leben gerufen). Auch der Bolognaprozess sieht Beteiligungsmöglichkeiten für Stakeholder vor. Im ,Europe du Savoir' soll der Wissende Zugang zu Entscheidungsprozessen erhalten, die ihm vorher verwehrt blieben. Wissen wird zur sozialen und politischen Ressource, und Expertenwissen nimmt einen zentralen Platz in der Gesellschaftsplanung ein - oder, mit den Worten Helmut Willkes: „Wissen im allgemeinen und Expertise als systematisiertes und organisiertes Wissen im besonderen verändern soziale Ordnung kontinuierlich, seit die Betonung der Verteilung und Verwendung von Wissen nicht mehr auf altem, traditionalem, unvordenklichem Wissen liegt, sondern auf neuem Wissen“ (Willke 1998: 241).

Die für Bologna typische unmittelbare Partizipation von Stakeholdern am Politikprozess könnte nun, im Sinne Ulrich Becks (1994), als alternative Version politischen Handelns verstanden werden, in der sich institutionalisierte Politikverflechtungen auflösen und Maßnahmen in einer offenen Akteursstruktur diskutiert werden. Erst diese Öffnung erlaube es, so Beck, mit den Ambivalenzen der risikodominierten Gesellschaft umzugehen und die ineffizienten Lösungsansätze traditioneller politischer Entscheidungsfindung zu überwinden. Mit anderen Worten: „The political breaks open and erupts beyond the formal responsibilities and hierarchies" (Beck 1994: 18). Nun aber stellt sich die Frage, inwiefern diese Vision tatsächlich eine Umsetzung findet: Was für eine Rolle nehmen die Stakeholder im Policy-Making-Prozess ein? Wie spiegelt sich das in der politischen Entscheidungsfindung wider? Kurzum: Wo liegen die Grenzen und Möglichkeiten des Inklusionskonzeptes Bolognas?

Am Beispiel der aktuellen Debatte um die Einführung der Meta-Akkreditierung im europäischen Hochschulraum soll der Einfluss der Stakeholder illustriert und im Lichte der oben formulierten Fragestellung analysiert werden. 


\section{Möglichkeiten und Grenzen der Realisierung eines wissensbasierten Gesellschaftsmodells}

Anlässlich der Berlin-Konferenz im Mai 2003 wurden die vier großen Stakeholder EUA, ESU, EURASHE und ENQA von den europäischen Bildungsministern beauftragt, konkrete Reformkonzepte zur Qualitätssicherung zu formulieren. Die so genannten „E4“ haben für die 2005 stattfindende Bergen-Konferenz zwei Policy-Texte verfasst, die eine tiefgreifende Restrukturierung des bestehenden Systems europäischer Qualitätssicherung vorsahen (ENQA 2005): Nationale und regionale Evaluations- und Akkreditierungsagenturen sollten von einer unparteiischen, supranationalen Kommission überprüft werden. Sollte ihre Eignung festgestellt werden, würde ihnen der Zugang zu einem Register zertifizierter Qualitätssicherungsagenturen gewährt werden. Die europäischen Hochschulen würden dann die Möglichkeit erhalten, eine beliebige Dienstleistungsagentur aus dem Register zu wählen, um ihre Studiengänge evaluieren oder akkreditieren zu lassen, ohne auf das (oftmals begrenzte) nationale Angebot an Agenturen zurückgreifen zu müssen. Mit der Einführung einer so genannten MetaAkkreditierung sollte eine Liberalisierung der nationalen Qualitätssicherung und ihrer Angebots- und Nachfragestruktur erreicht werden (Serrano-Velarde 2008). Die prinzipielle Gleichwertigkeit europäischer Qualitätssicherungsagenturen sollte dazu führen, nationale Marktbegrenzungen aufzulösen und ein europäisch ausgelegtes Netzwerk des Konsumentenschutzes zu begründen. Im Anbetracht der steigenden Studenten- und Arbeitskräftemobilität erklärte man die Internationalisierung des Konsumentenschutzes zur, conditio sine qua non' für die Realisierung eines integrierten europäischen Hochschulraums.

Nun sollte bedacht werden, dass Evaluation und Akkreditierung in Europa als Teil einer staatlichen Steuerungsreform im Hochschulbereich eingeführt wurden: Im Zuge einer umfangreichen Hochschulreform wurde den Evaluations- und Akkreditierungsagenturen die Verantwortung übertragen, Daten zur realen Hochschulleistung und eine Informationsgrundlage für Entscheidungsträger zu liefern (Neave 1988, 1998). Das europäische Reformprojekt, die nationale Hochschulsteuerung durch eine supranationale Qualitätskontrolle zu unterlaufen, mag auf den ersten Blick also verwirren: Wie kommt es dazu, dass ein intergouvernementales Reformprojekt Richtlinien zur Umgestaltung und Liberalisierung staatlicher Einflussbereiche bereitstellt? Die Antwort auf diese Frage führt uns zur komplizierten Politikstruktur des Bolognaprozesses zurück. Es ist den Stakeholdern zuzurechnen, dass die Meta-Akkreditierung überhaupt in die Politikdiskussion aufgenommen wurde. Sie sind es, die das ursprüngliche Registerkonzept der UNESCO (United Nations Educational, Scientific and Cultural Organization) und OECD (Organisation for Economic Co-operation and Development) aufgegriffen und an die Bildungsminister herangetragen haben.

\section{Ursprung und Entwicklung der Meta-Akkreditierung}

Evaluation und Akkreditierung haben sich seit Ende der 1980er Jahre zum lukrativen Unternehmen entwickelt, doch die Entdeckung illegaler Akkreditierungspraktiken begründete schon frühzeitig das internationale Interesse an Gesprächen zur Einführung eines übergeordneten Kontrollorgans. Als erste nichtstaatliche Organisation griff die International Association of University Presidents (IAUP) das Thema Meta-Akkreditierung auf und beauftragte den belgischen Bildungsexperten Dirk van Damme mit der Realisierung einer Studie zur Internationalisierung der Hochschulbildung und Akkreditierung. Van Dammes prominente Analysen aus den Jahren 1999 und 2001 (van Damme 1999, 2001) hoben die dramatischen Auswirkungen der Globalisierung des Hochschulbetriebs auf die bis dahin noch nationalstaatlich definierten Systeme des Konsumentenschutzes hervor. Demzufolge sei der wachsenden Unsicherheit hinsichtlich der Qualität nationaler und internationaler Hochschulangebote entgegenzuwirken, indem nationale Qualitätssicherungssysteme durch ein international aktives Kon- 
trollgremium ergänzt werden. Die Idee van Dammes, ein ,worldwide register for quality assurance and accreditation agencies" (van Damme 2001) ins Leben zu rufen, wurde kurze Zeit später von der UNESCO und der OECD aufgenommen, die seit 2001 an gemeinsamen Richtlinien zur „quality provision in cross-border higher education” arbeiteten (OCDE 2005). Der Fokus der UNESCO / OECD-Politikagenda auf den Konsumentenschutz im expandierenden, globalen Hochschulmarkt spiegelte die wachsende Sorge der Entwicklungsländer um die Qualität des importierten Hochschulangebotes wider. Zwar musste die Registeridee aus den 2005 ratifizierten ,lignes directrices“ aufgrund des scharfen Widerstands der Hochschulexporteure wieder gestrichen werden. Die langjährige Registerdebatte erweckte aber schon 2001 das Interesse der Generaldirektion Bildung und Kultur der europäischen Kommission, die kurz darauf den Punkt Meta-Akkreditierung für ihre bildungspolitische Strategie operationalisierte. Obwohl der Implementierungserfolg lange ausblieb, avancierte das Thema zum Leitmotiv der EU-Hochschulpolitik und führte zur Verfassung einiger kontrovers diskutierter Policy-Texte (European Commission 2004). Erst das Berliner Mandat 2003 bot der Kommission die Chance, die Registerfrage wieder aufzurollen und sie den Stakeholdern zuzuspielen. Schließlich bestanden bzw. bestehen privilegierte Kontakte zwischen den Stakeholdern der Mandatsgruppe (d.h. ESIB, EUA, EURASHE und ENQA) und deren wichtigstem Geldgeber, der europäischen Kommission.

Im Mai 2005 wurden die mandatierten Richtlinien zur Qualitätssicherung schließlich den Bildungsministern vorgestellt. Wie erwartet nahmen sie die Registeridee mit wenig Begeisterung auf und verweigerten die Ratifizierung. Einen Konfliktpunkt der damaligen Diskussion stellte insbesondere die Frage nach den Kompetenzen einer solchen Organisation und ihren Einflussmöglichkeiten auf nationale Belange dar (House of Commons 2007). Das ,enjeu national' der Qualitätssicherung, das an der Steuerungsfunktion von Evaluation und Akkreditierung festgemacht werden kann, verhinderte damals die Delegierung nationaler Regulierungskompetenzen an eine supranationale Instanz und setzte der Realisierung eines integrierten europäischen Evaluations- und Akkreditierungsmarktes, der von den Stakeholdern angestrebt wird, enge Grenzen. Zwei Jahre später, auf der interministeriellen Konferenz in London, scheint sich die Registeridee kaum weiterentwickelt zu haben. Bis auf eine Beteuerung guter Absichten weist das Kommuniqué keinerlei Spuren einer darüber hinaus gehenden Reflektion zur Umsetzung der Meta-Akkreditierung auf (London Communiqué 2007).

\section{Bologna und die politische Tradition der Industriegesellschaft}

Auch wenn Qualitätssicherung zum ,enjeu politique' europäischer Integrationsbestrebungen geworden ist, werden die Handlungsräume der Agenturen durch nationale Anforderungen und Erwartungen vorgegeben. Die Einführung der Meta-Akkreditierung, die den europäischen Hochschulen die freie Wahl ihres Akkreditierungs- bzw. Evaluationsanbieters zugesprochen hätte, scheiterte am notorischen Veto-Punkt (Tsebelis 2002) aller Europäisierungsbemühungen im Bildungsbereich: dem Subsidiaritätsprinzip. Das Inklusionsprinzip Bolognas weist also im Falle der pluralistischen Akteursstruktur durchaus schwerwiegende Verkürzungen auf. Die offene Politikstruktur ermöglicht zwar die Teilnahme einer wachsenden Zahl nichtstaatlicher Organisationen und Stakeholdergruppen am Policy-Prozess, doch beschränken sich deren Einflussmöglichkeiten auf die Politikformulierung. Eine Mitentscheidung bleibt, in einer von Nationalstaaten diktierten Interessensphäre, ausgeschlossen. Ungeklärt bleibt auch, inwiefern der Bolognaprozess seine strukturelle Offenheit bewahren kann. Derzeit stellt sich verstärkt das Problem einer möglichen Institutionalisierung des Policy-Prozesses, insbesondere seiner Follow-Up-Aktivitäten. Damit würde jedoch eine Inklusionsdynamik eingefroren werden, die sich innovationsfördernd auf den intergouvernementalen Reformprozess ausgewirkt hat. 
Sich auf die Ausführungen Ulrich Becks beziehend könnte man sich nun die Frage stellen, ob alternative Formen politischer Entscheidungsfindung unter diesen Umständen realisiert werden können. Trotz Inklusionsbestrebungen scheint die Policy-Struktur des Bolognaprozesses die hierarchischen Beziehungen zwischen den involvierten Parteien unberührt zu lassen. Kurzum, die Policy-Struktur des Bolognaprozesses orientiert sich an einem Grundprinzip, das laut Ulrich Beck auf industriegesellschaftliche Politiktraditionen zurückzuführen ist, nicht aber auf ein wissensbasiertes und integriertes Gesellschaftsmodell schließen lässt: „Politics in the nation-state structure and rule system is no departure into a new land of the political and geopolitical or the global risk society“ (Beck 1994: 37).

\section{Schluss}

Die dem Bolognaprozess zugrunde liegende Vision der Wissensgesellschaft wurde an einer dreidimensionalen Inklusionsdynamik festgemacht:

1. Der Bolognaprozess wurde zunächst als Reformversuch gedeutet, breite Bevölkerungsschichten in ein wissensdominiertes Gesellschaftsmodell zu integrieren. Der europäische Policy-Prozess ist dem Prinzip nach eine Wissenspolitik, die darauf abzielt, die Erwerbsmöglichkeiten von Wissen zu erweitern. Dies soll über die Regulierung und Rationalisierung von Wissensprozessen erreicht werden.

2. Der Bolognaprozess kann aber auch als neue Bildungsphilosophie verstanden werden, die das Ausbildungsmoment nicht länger als zeitlich begrenztes Phänomen begreift. Vielmehr behält das Individuum sein ganzes Leben lang die Abhängigkeit zum Bildungssystem bei. Die Inklusionsdynamik greift also bis in die individuelle Lebensführung hinein, die sich dem wissensgesellschaftlichen Credo ,lebenslangen Lernens' verschreiben muss.

3. Der Bolognaprozess hat schließlich eine bildungspolitische Plattform ins Leben gerufen, im Rahmen derer relevante Stakeholdergruppen in den Policy-Making-Prozess eingeschlossen werden. Als Interessenvertreter und Bildungsexperten sind die Stakeholdergruppen maßgeblich an der Erstellung der Reformvorschläge und Policy-Erklärungen beteiligt.

Bei der letztgenannten Dimension ließen sich jedoch Inkohärenzen und inhaltliche Verkürzungen in der Umsetzung der Inklusionsstrategien empirisch nachweisen: In der Tat bleibt der Einfluss der Interessenvertreter auf die Vorbereitung der Konferenzen und die Redaktion der Policy-Dokumentation beschränkt. Von einer Mitbestimmung am Entscheidungsprozess kann keine Rede sein. So werden auch die fortschrittlichsten Politikideen von einem Politikprozess unterlaufen, welcher die offene Akteursstruktur der Politikformulierung vom staatlich monopolisierten Entscheidungssystem abkoppelt. Inwiefern aber wirkt sich ein solcher Determinismus letzten Endes auch auf die Politikformulierung aus? Nicht nur die Politikstruktur ist einem Rationalisierungsdruck ausgesetzt. Auch die Policy-Inhalte laufen Gefahr, einem falsch verstandenen Pragmatismus anheim zu fallen. Seit 2003 sind Veränderungen im Diskurs und Inhalt der interministeriellen Erklärungen festzustellen. Die bildungsphilosophische und gesellschaftspolitische Untermauerung des Reformansatzes früher Policy-Papiere weicht immer mehr einer rationalisierten, ja technokratisch anmutenden Aufzählung implementierungsbereiter Reformkonzepte. Ausführungen zur gesellschaftlichen Vision, die dem Projekt zur Realisierung einer „European Higher Education Area“ vorausgingen, werden zwar wiederholt. Inhaltlich finden allerdings kaum Entwicklungen statt. Der Erfolg des Bolognaprozesses wird an seiner Expansionsrate, aber auch an der Implementierung der einzelnen Reformpunkte gemessen, nicht aber vor dem Hintergrund jener gesellschaftspolitischen Vision diskutiert, auf der das Projekt zur Realisierung eine integrierten europäischen Hochschulraumes, eines ,Europe du Savoir', ursprünglich zurückzuführen ist. 


\section{Anmerkungen}

I Den Prozess der Übersetzung bestimmter Begriffe in neue Kontextzusammenhänge definieren Sabine Maasen und Peter Weingart (1995) z.B. als „dynamics of knowledge“. In ihrem Forschungsprogramm, das sich der Rekonstruktion diskursiver Diffusionsprozesse widmet, gehen die Autoren der Frage nach, wie sich die Bedeutung bestimmter Sinneinheiten (z.B. Metaphern) verschieben, stabilisieren oder aber zerlaufen. Der analytische Fokus des Forschungsansatzes liegt auf dem interaktiven Diffusionsprozess als eigentliches Evolutionsmoment von Wissen. Die vorliegende Studie zielt jedoch nicht darauf ab, die begriffsgeschichtliche „Karriere“ (Maasen / Weingart 2000) der „Wissensgesellschaft“ im europäischen Bildungsdiskurs nachzuzeichnen. Der Beitrag handelt vielmehr von einem bildungspolitischen Entwurf der Wissensgesellschaft, den es zu durchleuchten, auseinanderzunehmen und auf seine Implikationen hin zu prüfen gilt.

II In der wissensbasierten Ökonomie geht es um neue Formen der Wissensproduktion: Nico Stehr, z.B., interpretiert Wissen als wichtigste Ursache wirtschaftlichen Wachstums neben den klassischen Faktoren Kapital und Arbeit: „In der Wissensgesellschaft machen kognitive Faktoren, Kreativität, Wissen und Information in zunehmendem Maße den Großteil des Wohlstands eines Unternehmens aus. Auf den Punkt gebracht bedeutet dies, dass in den Wirtschaften dieser Länder für die Produktion von Gütern und Dienstleistungen [...] andere Faktoren im Mittelpunkt stehen als ,the amount of labor time or the amount of physical capital'" (Stehr 1994: 35 f). Auch Peter Drucker, Daniel Bell und Manuel Castells gründen ihre Ansätze auf der Identifikation neuer Produktivkräfte und neuer Prinzipien der Wertschöpfung, bei denen Wissen, Innovation und technische Entwicklung (im Gegensatz zu Arbeit, Kapital, Land Maschinen oder Energie) die entscheidende Rolle spielen (Steinbicker 2001). Dieses neue Verständnis der Wertschöpfung lässt sich auch in den bildungspolitischen Diskursen wiederfinden (siehe hierzu Ausführungen in Punkt 3).

III Aletta Norval thematisiert in „The Things we do with Words - Contemporary Approaches to the Analysis of Ideology“ (Norval 2000) die rhetorische bzw. diskursive Wende in der neueren Ideologieforschung (,rethorization of ideology“): Das traditionelle Ideologieverständnis, das sich an der Dualität von Wahrheit / Wissen versus Falschheit orientierte, sei unter Umständen, in denen Wahrheit und Wissen selbst von Unsicherheit und Ambivalenz befallen sind, nicht mehr anwendbar. Neuere Ansätze der Ideologieforschung gehen deshalb von einer breiten Ideologiedefinition aus, die sich an die Diskurstheorie anlehnt. Als Ideologie bezeichnet werden handlungsleitende Vorannahmen, Repräsentationen und Diskurse, die einen Stabilitätsgrad erreicht haben (also nicht mehr hinterfragt werden). Ideologie wird infolgedessen zum allgegenwärtigen Phänomen, und Ziel der Ideologieforschung ist es, den Prozess nachzuvollziehen, der zur Institutionalisierung von Ideen und Diskursen führt: „It aims to investigate the extent to which ideologies can be fruitfully understood as combinations of decontested, or naturalized, conceptual formations, practices and images for identification" (Norval 2000: 316).

IV Mit Integration beschreibt Niklas Luhmann (1995) die Koppelung differenzierter Teilsysteme. Der Begriff der Integration beschreibt also eine prinzipiell systemexterne Dynamik.

Die Unterscheidung Inklusion / Exklusion hingegen ist eine systeminterne Unterscheidung. Mit dem Modus der Inklusion beschreibt ein soziales System das, was es als Teilnahmebedingungen setzt bzw. als Teilnahmechancen in Aussicht stellt. Es sollte angemerkt werden, dass der Inklusionsprozess Bolognas nicht vollständig in der systemtheoretischen Definition aufgeht, insofern sich das Reformprojekt als gesellschaftsumspannendes Paradigma versteht, d.h. die Inklusion eines größtmöglichen Bevölkerungsanteils angestrebt wird. 
v Mit der Ausweitung des Lernprozesses auf die gesamte Lebensspanne eines Individuums wird deutlich, dass sich die ,Mitgliedschaft' im Teilsystem nicht länger auf einen Lebensabschnitt reduzieren lässt. Die zeitliche Expansion der, Mitgliedschaft' kann als Inklusionsprozess beschrieben werden, insofern das Individuum sein Leben lang in einem Abhängigkeitsverhältnis zum Bildungssystem steht.

VI Nico Stehr definiert Wissenspolitik vornehmlich als ,,regulative, restriktive Politik; d.h. es geht um die politische Handlung, die außerhalb des Wissenschaftssystems initiiert wird und die auf eine Kontrolle der praktischen Verwendung neuer Erkenntnisse zielt (Stehr 2003: 87)“. Die Entwicklung des Politikfeldes Wissenspolitik ist demnach eine verzögerte Reaktion auf die außerordentliche Geschwindigkeit mit der neue Erkenntnisse und technische Möglichkeiten in modernen Gesellschaften wachsen: „Die moderne Gesellschaft lässt sich als eine Wissensgesellschaft beschreiben, weil ein wachsender Teil der gesellschaftlichen Handlungsräume und Handlungsabfolgen von Erkenntnissen mitbestimmt und durchdrungen ist" (Stehr 2003: 19f). Nico Stehrs Forschungsinteresse betrifft vornehmlich Fragen der Wissensgenerierung, der Überwachung und Kontrolle neuer Erkenntnisse. Der Begriff der Wissenspolitik wirkt also recht verkürzend, vergleicht man ihn mit dem weitaus breiter angelegten Wissensbegriff. Als modernes Organisations- und Integrationsprinzip verlangt Wissen aber nach einer multidimensional ausgerichteten Definition von Wissenspolitik als Regulierung von Wissensgenerierung, -diffusion und -erwerb. In dieser Hinsicht sollte der Bolognaprozess als Bestandteil einer europäisch angelegten Wissenspolitik angesehen werden, die darauf abzielt, den Zugang und die Wiederverwertungsmöglichkeit der Wissensressource systematisch zu regulieren.

VII Die Expertise der Stakeholder ergibt sich nicht nur aus der Konzentrierung des so genannten Nutzerwissens. Die meisten Stakeholder verfügen auch über aktive, think-tanks', in denen sich ehemalige Akademiker für die Politikberatung einsetzen. Die Handlungsfähigkeit einer Gruppe hängt jedoch von ihren finanziellen Ressourcen ab, die sie zum Teil über Mitgliederbeiträge gewinnt. Umfangreiche Mitgliederorganisationen wie z.B. EUA (European University Association) verfügen über ausreichend Mittel, um ganze Arbeitsgruppen und Projektförderlinien ins Leben zu rufen. Dagegen kann sich ihre Schwesterorganisation EURASHE (die European Association of Institutions in Higher Education) kaum mehr als zwei volle Mitarbeiterstellen leisten. Dementsprechend lässt sich auch der Einfluss der Stakeholdergruppen auf den Politikprozess relativieren. 


\section{Literatur}

Beck, U. (1994): The Reinvention of Politics. Towards a Theory of Reflexive Modernization, in: U. Beck / A. Giddens / S. Lash (Hrsg.), Reflexive Modernization, Cambridge / Oxford, S. 1-56.

Bell, D. (1976): The Coming of Post-Industrial Society. A Venture in Social Forecasting, New York.

Bittlingmayer, U. (2005): „Wissensgesellschaft“ als Wille und Vorstellung, Konstanz.

Bologna Declaration (1999): The Bologna Declaration of 19 June 1999. Joint Declaration of the European Ministers of Education, abgerufen im Mai 2004 unter http://www.bologna-bergen2005.no.

Castells, M. (1996): La société en réseaux. L'ère de l'information, Paris.

ENQA (2005): Standards and Guidelines for Quality Assurance in the European Higher Education Area, Helsinki, abgerufen im Juli 2005 unter http://www.enqa.eu.

Etzkowitz, H. / L. Leydesdorff (Hrsg.)(1997): Universities and the Global Knowledge Economy : A Triple Helix of University-Industry-Government Relations, London.

European Commission (2004): Proposal for a Recommendation of the Council and of the European Parliament on Further European Cooperation in Quality Assurance in Higher Education, Ref: COM (2004) 642 final, 12. Oktober 2004, abgerufen im Dezember 2005 unter http://europa.eu.int.

Europäischer Rat (2000): Europäischer Rat 23. und 24. März in Lissabon. Schlussfolgerungen des Vorsitzes, abgerufen im Dezember 2005 unter http://europa.eu.int.

Froschauer, U. / M. Lueger (2003): Das qualitative Interview. Zur Praxis interpretativer Analyse sozialer Systeme, Wien.

Fuhrmann, M. (2002): Bildung: Europas kulturelle Identität, Stuttgart.

Gibbons, M. / C. Limoges / H. Nowotny / S. Schwartzman / P. Scott / M. Trow (Hrsg.)(1994): The New Production of Knowledge. The Dynamics of Science and Research in Contemporary Societies, London.

Heidenreich, M. (2003): Die Debatte um die Wissensgesellschaft. in: S. Böschen / I. Schulz-Schaeffer (Hrsg.), Wissenschaft in der Wissensgesellschaft, Opladen, S. 25-55.

House of Commons (2007): The Bologna Process. Government Response to the Committee's Fourth Report of Session 2006-2007, London.

Kok, W. (2004): Facing the Challenge. The Lisbon Strategy for Growth and Employment, Report from the High Level Group Chaired by Wim Kok, abgerufen im März 2005 unter http://europa.eu.int.

Kübler, H.D. (2004): Mythos Wissensgesellschaft. Gesellschaftlicher Wandel zwischen Information, Medien und Wissen, Wiesbaden.

London Communiqué (2007): Towards the European Higher Education Area. Responding to Challenges in a Globalised World. Communique of the Conference of Ministers Responsible for Higher Education in London on 18 May 2007, abgerufen im Mai 2007 unter http://www.ond.vlaanderen.be.

Luhmann, N. (1995): Inklusion und Exklusion. In: Niklas Luhmann, Soziologische Aufklärung 6, Opladen: Westdeutscher Verlag, S. 237-264.

Maasen, S. / P. Weingart (1995): Metaphors - Messengers of Meaning. A Contribution to an Evolutionary Sociology of Science, in: Science Communication 17 / 9, S. 9-31.

Maasen, S. / P. Weingart (2000): Metaphors and the Dynamics of Knowledge, London.

Muller, P. (1990): Les Politiques Publiques, Paris.

Neave, G. (1988): On the Cultivation of Quality, Efficiency and Enterprise. An Overview of Recent Trends in Higher Education in Western Europe 1986-1988, in: European Journal for Education 23, S. 7-23.

Neave, G. (1998): The Evaluative State Reconsidered, in: European Journal of Education 33 / 3, S. 265-284.

Norval, A. (2000): The Things We Do With Words. Contemporary Approaches to the Analysis of Ideology, in: British Journal of Political Sciences 30 / 2, S. 313-346. 
OCDE (2005): Lignes directrices pour des prestations de qualité dans l'enseignement supérieur transfrontalier, Paris.

Serrano-Velarde, K. (2008): Evaluation, Accreditation and Politics. Why We are still Far Away from an Integrated European Market of Quality Assurance Agencies, in: Higher Education Management and Policy $20 / 3$, S. 1-18.

Sevraes, J. (Hrsg.)(2003): The European Information Society. A Reality Check, Bristol.

Sorbonne Joint Declaration (1998): Joint Declaration on Harmonisation of the Architecture of the European Higher Education Systems by the Four Ministers in Charge for France, Germany, Italy and the United Kingdom, Paris, 25. Mai 1998, abgerufen im Mai 2004 unter http://www.bologna-bergen2005.no.

Stehr, N. (1994): Knowledge Societies. London.

Stehr, N. (2003): Wissenspolitik. Die Überwachung des Wissens, Frankfurt / Main.

Steinbicker, J. (2001): Zur Theorie der Informationsgesellschaft. Ein Vergleich der Ansätze von Peter Drucker, Daniel Bell und Manuel Castells, Opladen.

Tsebelis, G. (2002): Veto Players. How Institutions Work, New York.

Van Damme, D. (1999): Internationalisation in Quality Assurance. Towards Worldwide Accreditation? Auftragsstudie für die XII. IAUP Konferenz in Brüssel vom 11.-14. Juli 1999, abgerufen im März 2003 unter http://www.ia-up.org.

Van Damme, D. (2001): Towards a Worldwide Quality Register for Quality Assurance Agencies. Auftragsstudie für das IAUP Meeting in Gent vom 13.-15. Dezember 2001, abgerufen im März 2003 unter http://www.ia-up.org.

Webster, F. / K. Robbins (1989): Plan and Control. Towards a Cultural History of the Information Society, in: Theory and Society 18, S. 323-351.

Webster, F. (2002): Theories of the Information Society, London.

Willke, H. (1997): Supervision des Staates. Frankfurt / Main.

Willke, H. (1998): Systemisches Wissensmanagement, Stuttgart.

Willke, H. (2007): Smart Governance: Governing the Global Knowledge Society. Frankfurt Main / New York.

\author{
Jun.-Prof. Dr. Kathia Serrano-Velarde \\ Institut für Soziologie \\ Universität Heidelberg \\ Bergheimer Str. 58 \\ 69115 Heidelberg \\ kathia.serrano@soziologie.uni-heidelberg.de
}

